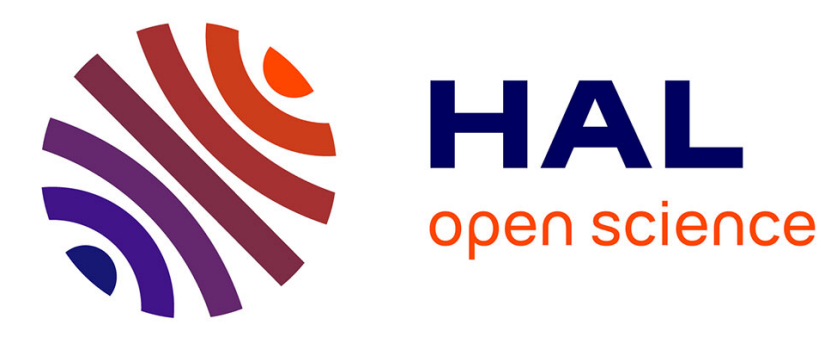

\title{
Petri Net Discovery of Discrete Event Processes by Computing T-invariants
}

\author{
Tonatiuh Tapia-Flores, Ernesto López-Mellado, Ana-Paula Estrada-Vargas, \\ Jean-Jacques Lesage
}

\section{- To cite this version:}

Tonatiuh Tapia-Flores, Ernesto López-Mellado, Ana-Paula Estrada-Vargas, Jean-Jacques Lesage. Petri Net Discovery of Discrete Event Processes by Computing T-invariants. 19th IEEE Int. Conf. on Emerging Technologies and Factory Automation (ETFA'14), Sep 2014, Barcelone, Spain. paper 345,8 p. hal-01067790

\section{HAL Id: hal-01067790 https://hal.science/hal-01067790}

Submitted on 24 Sep 2014

HAL is a multi-disciplinary open access archive for the deposit and dissemination of scientific research documents, whether they are published or not. The documents may come from teaching and research institutions in France or abroad, or from public or private research centers.
L'archive ouverte pluridisciplinaire HAL, est destinée au dépôt et à la diffusion de documents scientifiques de niveau recherche, publiés ou non, émanant des établissements d'enseignement et de recherche français ou étrangers, des laboratoires publics ou privés. 


\title{
Petri Net Discovery of Discrete Event Processes by Computing T-invariants
}

\author{
Tonatiuh Tapia-Flores ${ }^{1}$, Ernesto López-Mellado ${ }^{1}$, Ana Paula Estrada-Vargas ${ }^{2}$, Jean-Jacques Lesage ${ }^{3}$ \\ ${ }^{1}$ CINVESTAV Unidad Guadalajara. Av. del Bosque 1145, Col. El Bajío. 45019 Zapopan, Mexico \\ ${ }^{2}$ Oracle de México S. A. de C. V. Av. de los Empresarios 135, Col. Puerta de Hierro. 45110 Zapopan, Mexico \\ ${ }^{3}$ LURPA, Ecole Normale Supérieure de Cachan. 61, Av. du Président Wilson. 94235 Cachan Cedex, France \\ \{ttapia, elopez\}@gdl.cinvestav.mx, ana.estrada@oracle.com, Jean-Jacques.lesage@lurpa.ens-cachan.fr
}

\begin{abstract}
In this paper the problem of discovering a Petri net $(P N)$ from sampled events sequences representing the execution of industrial or business processes is addressed. A method for building a 1-bounded PN from a single event sequence $S$ composed of numerous execution traces is presented; it is based on determining causal and concurrency relations between tasks. A technique for computing the t-invariants of the $P N$ from $S$ is proposed; the obtained invariants allow determining the structure of a PN that executes $S$. The algorithms derived from the method have been implemented and tested on numerous examples of diverse complexity.
\end{abstract}

\section{Keywords-Model discovery; Petri Nets; t-invariants}

\section{INTRODUCTION}

The synthesis of formal models from external observation of systems behaviour is an interesting and challenging approach for reverse engineering purposes in discrete event systems. Although the problem is relatively recent, it deserves the attention of several research groups in the fields of discrete event systems (DES) and workflow management systems (WMS).

Pioneer works on the matter, named language learning techniques, appear in computer sciences. The aim was to build fine representations (finite automata, grammars) of languages from samples of accepted words [1,2].

In the field of DES, where the problem is named identification, several approaches have been proposed for building models representing the observed behaviour of automated processes. The incremental approach proposed in $[3,4]$ allows building safe interpreted Petri net (PN) models from a continuous stream of system's outputs. In [5], a method based on the statement and solution of an integer linear programming problem is proposed; it allows building PN from a set of sequences of events. Extensions of this method are proposed in [6, 7]. In [8] a method for deriving finite automata from sequences of inputs and outputs is presented; it is applied to fault detection of manufacturing processes. An extension to this method that allows obtaining distributed system models is presented in [9]. In [10] Input-output identification of automated manufacturing process is addressed; an interpreted $\mathrm{PN}$ is obtained from a set of sequences of input-output vectors collected from the controller during the system cyclic operation. The method is extended for dealing with a long single observation of input-output vectors [11]. More complete reviews on DES identification can be found in [12] and [13].

In WMS the analogous problem is named workflow mining; the system observation is given as a set of sequences from a finite alphabet of tasks, representing execution logs of business processes. A first proposal is reported in [14], in which a finite automaton, called conformal graph is obtained. In [15] it is proposed a probabilistic approach to find the concurrent and direct relations between tasks. The input of the method is a sequence of events that represent the activities that have occurred in a workflow management system; the obtained model is graph similar to a PN. In [16] a mining method called algorithm alpha is presented. In this method a workflow tasks log composed by several traces is recorded sequentially and processed yielding a subclass of PN called workflow net. Numerous publications present extensions of this algorithm namely [17, 18, and 19]. In particular in this last work a strong hypothesis is held: the workflow engine provides, for every task in the log, the next tasks to be executed even if they are not consecutive; this means that all the causal relationships are a priori known. More related works can be found in [20].

In the present paper a new method for building a safe Petri net (PN) from a single sequence of tasks $\mathrm{S}$, composed by numerous processes execution traces, is proposed. It follows the approach presented in [10] and proposes new results allowing addressing more complex behaviours such as implicit dependencies between tasks that are not observed consecutively. The method is based on determining, from S, causal and concurrency relations between tasks and the computing of the t-invariants of the PN to discover. The obtained invariants allow, first, determining the initial structure of a PN, and later, adjusting the model when the computed t-invariants do not coincide with those of the initial model. The paper is organized as follows. In Section 2, the basic notions on PN are recalled. Section 3 states the addressed problem. In Section 4, basic relations, computed form the tasks sequence, are introduced. Section 5 presents a technique for determining the t-invariants. In Section 6, the PN synthesis method is described. Section 7 outlines implementation and tests.

\section{BACKGROUND}

This section presents the basic concepts and notations of ordinary PN used in this paper. 
Definition 1. An ordinary Petri Net structure $G$ is a bipartite digraph represented by the 4-tuple $G=(P, T, I, O)$ where: $P=\left\{p_{1}, p_{2}, \ldots, p_{|P|}\right\}$ and $T=\left\{t_{1}, t_{2}, \ldots, t_{|T|}\right\}$ are finite sets of vertices named places and transitions respectively; $I(O): P \times T \rightarrow\{0,1\}$ is a function representing the arcs going from places to transitions (from transitions to places).

The incidence matrix of $G$ is $C=C^{+}-C^{-}$, where $C^{-}=\left[c_{i j}{ }^{-}\right] ; c_{i j}{ }^{-}=I\left(p_{i}, t_{j}\right)$; and $C^{+}=\left[c_{i j}{ }^{+}\right] ; c_{i j}{ }^{+}=O\left(p_{i}, t_{j}\right)$ are the pre-incidence and post-incidence matrices respectively.

A marking function $M: P \rightarrow Z^{+}$represents the number of tokens residing inside each place; it is usually expressed as an $|P|$-entry vector. $Z^{+}$is the set of nonnegative integers.

Definition 2. A Petri Net system or Petri Net (PN) is the pair $N=\left(G, M_{0}\right)$, where $G$ is a PN structure and $M_{0}$ is an initial marking.

In a PN system, a transition $t_{j}$ is enabled at marking $M_{k}$ if $\forall p_{i} \in P, M_{k}\left(p_{i}\right) \geq I\left(p_{i}, t_{j}\right)$; an enabled transition $t_{j}$ can be fired reaching a new marking $M_{k+1}$, which can be computed as $M_{k+1}=M_{k}+C u_{k}$, where $u_{k}(i)=0, \mathrm{i} \neq \mathrm{j}, u_{k}(\mathrm{j})=1$; this equation is called the PN state equation. The reachability set of a PN is the set of all possible reachable markings from $M_{0}$ firing only enabled transitions; this set is denoted by $R\left(G, M_{0}\right)$.

Definition 3. A t-invariant $Y_{i}$ of a $P N$ is an integer solution to the equation $C Y_{i}=0$ such that $Y_{i} \geq 0$ and $Y_{i} \neq 0$. The support of $Y_{i}$ denoted as $\left\langle Y_{i}\right\rangle$ is the set of transitions whose corresponding entries in $Y_{i}$ are strictly positive. $Y$ is minimal if its support is not included in the support of other t-invariant. A t-component $G\left(Y_{i}\right)$ is a subnet of $P N$ induced by a $\left\langle\mathrm{Y}_{i}\right\rangle$ : $\mathrm{G}\left(Y_{i}\right)=\left(P_{i}, T_{i}, I_{i}, O_{i}\right)$, where $\mathrm{P}_{\mathrm{i}}=^{\bullet}\left\langle Y_{i}\right\rangle \cup\left\langle Y_{i}\right\rangle^{\bullet} ; \mathrm{T}_{\mathrm{i}}=\left\langle Y_{i}\right\rangle, I_{i}=$ $P_{i} \times T_{i} \cap I$, and $O_{i}=P_{i} \times T_{i} \cap O$; where ${ }^{\bullet} \Theta\left(\Theta^{*}\right)$ is the set formed by the input (output) nodes to (from) nodes in $\Theta$.

\section{PROBLEM STATEMENT AND PROPOSED APPROACH}

\section{A. Model discovery}

First, we formulate the problem of model discovering in a general way; afterwards this technique is placed in the contexts of automated manufacturing processes and workflow management systems.

Definition 4. Given a finite alphabet of events or tasks $T=\left\{t_{1}, t_{2}, \ldots, t_{n}\right\}$ and a set of finite sequences $S_{i}=t_{1} t_{2} \ldots t_{j} \in T^{*}$, we define the PN discovery problem as the synthesis of a 1bounded PN structure using only transitions in $T$ and the discovery of an initial marking, which allows firing every $S_{i}$. The number of places of the $\mathrm{PN}$ is not known a priori.

In the context of automated manufacturing systems, $S_{i}$ represents the observation of relevant input-output events sampled from the controller during a long execution period of time, for example a complete production process performing repetitive jobs [10].

In the context of workflow mining, the observed behaviour is a $\log$ composed by traces $\sigma_{i} \in T^{*}$, which are sampled from the beginning to the end of execution traces (cases). In the current problem formulation a $S$ can be formed by the concatenation of tasks traces $S=\sigma_{1} \sigma_{2} \ldots \sigma_{r}$ regardless the order of $\sigma_{i}$ in $S$. The knowledge of cases delimiting, i.e., the beginning and ending of traces, is no longer required.

Assumptions. In both contexts it is assumed that processes are well behaved, i.e. there are no faults, deadlocks, or overflows during the observation period. This is a realistic assumption since the processes whose models have to be discovered are supposed to be in operation, although the model is currently unknown or ill known. Thus we can consider that the event stream $S \in T^{*}$ is generated by a deadlock-free 1-bounded PN to be discovered.

\section{B. Overview of the method}

The proposed method synthesises an ordinary PN structure and finds an initial marking from which $S$ can be fired. It focuses on the computation of the causal and concurrent relations between the tasks in the sequence $S$. This is achieved by determining the t-invariants (that are supposed to exist since most systems exhibit repetitive behaviour), which also are used to find causal implicit relations between events that are not observed consecutively.

In a first stage several binary relations between transitions are determined from $S$; based on these relations the tinvariants are computed. Afterwards, causal and concurrent relations are determined, and together with the discovered tinvariants, the structure of a PN model is built. Finally, again the t-invariants are used for reducing the possible exceeding language by determining causality between events not observed consecutively. The method is presented for dealing with a single sequence $S$ since the extension to deal with several $S_{i}$ is straightforward.

\section{BASIC CONCEPTS AND RELATIONS}

First we introduce several relations derived directly from $S$. Some of the following definitions have been taken and adapted from [10].

Definition 5. The relationship between transitions that are observed consecutively in $S$ is expressed in the relation $S e q \subseteq$ $T \times T$ which is defined as $\operatorname{Seq}=\left\{\left(t_{j}, t_{j+1}\right)|1 \leq j<| S \mid\right\} ; t_{a}$ Seq $t_{b}$ will be frequently denoted as $t_{a}<t_{b}$. The relation between transitions that never occur consecutively in $S$ is $T \times T \mathrm{Neq}$; pairs in this relation are denoted as $t_{a}><t_{b}$.

Definition 6. Every couple of consecutive transitions $\left(t_{a}, t_{b}\right)$ $\in S e q$ can be classed into one of the following situations: i) Causal relationship. The occurrence of $t_{a}$ enables $t_{b}$, denoted as $\left[t_{a}, t_{b}\right]$. In a PN structure, this implies that there must be at least one place from $t_{a}$ to $t_{b}$. ii) Concurrent relationship. If both $t_{a}$ and $t_{b}$ are simultaneously enabled, and $t_{a}$ occurs first, its firing does not disable $t_{b}$. In a PN structure this implies that it is impossible the existence of a place from $t_{a}$ to $t_{b}$. In this case, $t_{a}$ and $t_{b}$ are said to be concurrent, denoted as $t_{a} \| t_{b}$.

Now a relation that establishes a key property named repetitive dependency is introduced.

Definition 7. A transition $t_{j}$ is repetitively dependent of $t_{k}$, denoted as $t_{j}<t_{k}$ iff $t_{k}$ is always observed between two apparitions of $t_{j}$ in $S$. If $t_{j}$ has been observed at least twice in $S$, 
then $t_{j}<t_{j}$. The set of transitions from which $t_{j}$ is repetitively dependent is given by the function $R d\left(t_{j}\right): T \rightarrow 2^{T}$; then $\operatorname{Rd}\left(t_{j}\right)=$ $\left\{t_{k} \mid t_{j}<t_{k}\right\}$. If $t_{j}$ was observed only once in $S$, then $R d\left(t_{j}\right)=\varnothing$.

Property 1. The transitions in a $R d\left(t_{j}\right)$ are included in the support of at least one t-invariant.

Proof. $R d\left(t_{j}\right)$ is the set of transitions that must invariantly occur to fire $t_{j}$ repeatedly. Thus the proof follows directly from Definition 7 and the concept of t-invariant. Any $t_{k} \in R d\left(t_{j}\right)$ may belong also to other t-components.

Example 1. Consider the set of tasks $T=\left\{t_{1}, t_{2}, t_{3}, t_{4}, t_{5}, t_{6}\right.$, $\left.t_{7}\right\}$ and the sequence $S=\boldsymbol{t}_{\mathbf{1}} t_{2} t_{3} t_{4} \boldsymbol{t}_{\mathbf{1}} t_{2} t_{4} t_{3} t_{5} t_{6} t_{7} t_{4} \boldsymbol{t}_{1} t_{2} t_{3} t_{4} t_{5} t_{6}$ $t_{7} t_{4} \boldsymbol{t}_{\mathbf{1}} t_{2} t_{3} t_{4} \boldsymbol{t}_{\mathbf{1}} t_{2} t_{3} t_{4} t_{5} t_{6} t_{7} t_{4} t_{5} t_{6} t_{7} t_{4} \boldsymbol{t}_{\mathbf{1}} t_{2} t_{3} t_{4} t_{5} t_{6} t_{7} t_{4} t_{5} t_{6} t_{7}$ $t_{4} t_{5} t_{6} t_{7} t_{4} t_{5} t_{6} t_{7} t_{4} t_{5} t_{6} t_{7} t_{4} \boldsymbol{t}_{\mathbf{1}} t_{2} t_{4} t_{3} t_{1} t_{2} t_{3} t_{4} \boldsymbol{t}_{\mathbf{1}} t_{2} t_{3} t_{4} \boldsymbol{t}_{\mathbf{1}} t_{2} t_{4} t_{3}$ $\boldsymbol{t}_{\mathbf{1}} t_{2} t_{3} t_{4} \boldsymbol{t}_{\mathbf{1}}$. From $S$ it is obtained $\operatorname{Seq}=\left\{\left(t_{1}, t_{2}\right),\left(t_{2}, t_{3}\right),\left(t_{3}, t_{4}\right)\right.$, $\left(t_{4}, t_{1}\right),\left(t_{4}, t_{5}\right),\left(t_{5}, t_{6}\right),\left(t_{6}, t_{7}\right),\left(t_{7}, t_{4}\right),\left(t_{2}, t_{4}\right),\left(t_{4}, t_{3}\right),\left(t_{3}, t_{1}\right),\left(t_{3}\right.$, $\left.\left.t_{5}\right)\right\}$. Furthermore, one may observe that $t_{1}<t_{2}, t_{1}<t_{3}, t_{1}<t_{4}$, thus $R d\left(t_{1}\right)=\left\{t_{1}, t_{2}, t_{3}, t_{4}\right\}$. The rest of the $R d$ sets are $R d\left(t_{2}\right)=\left\{t_{1}, t_{2}\right.$, $\left.t_{3}, t_{4}\right\}, \operatorname{Rd}\left(t_{3}\right)=\left\{t_{1}, t_{2}, t_{3}\right\}, \operatorname{Rd}\left(t_{4}\right)=\left\{t_{4}\right\}, \operatorname{Rd}\left(t_{5}\right)=\left\{t_{4}, t_{5}, t_{6}, t_{7}\right\}$, $R d\left(t_{6}\right)=\left\{t_{4}, t_{5}, t_{6}, t_{7}\right\}, R d\left(t_{7}\right)=\left\{t_{4}, t_{5}, t_{6}, t_{7}\right\}$.

Definition 8. Two transitions $t_{a}, t_{b}$ are called transitions in a two-length cycle $(T c)$ relation if $S$ contains the subsequences $t_{\mathrm{a}} t_{\mathrm{b}} t_{\mathrm{a}}$ or $t_{\mathrm{b}} t_{\mathrm{a}} t_{\mathrm{b}}$. The set of transition pairs fulfilling this feature is denoted by $T c$. When the subsequence $t_{a} t_{a}$ appears in $\mathrm{S}, t_{a}$ is in the relation named self-loop $(s l)$.

It is easy to see that simple substructures of PN can be derived straightforward from $T c$ or sl. From Example 1, $T c=s l=\varnothing$.

Now, conditions for determining causal and concurrency relationships are given.

Proposition 1. Let $t_{a}, t_{b}$ be two transitions in $T$; then $t_{a} \| t_{b}$ if $\left(\mathrm{t}_{\mathrm{a}}, \mathrm{t}_{\mathrm{b}}\right), \quad\left(t_{b}, t_{a}\right) \in S e q$, i.e. $t_{a}$ and $t_{b}$ have been observed consecutively in $\mathrm{S}$ in both orders, and if $t_{a}, t_{b}$ do not form a $T c$.

Proof. It follows from Definition 6(ii) and from the condition that excludes the subsequence characterising a $T c$.

Thus, the set of concurrent transition pairs deduced from $S$ is ConcR $=\left\{\left(t_{a}, t_{b}\right) \mid t_{a}<t_{b} \wedge t_{b}<t_{a} \wedge\left(t_{a}, t_{b} \notin T c\right)\right\}$. Notice that this is a symmetric relation.

Proposition 2. Let $t_{a}, t_{b}$ be two transitions in $T$ such that $t_{a}<t_{b}$; then $\left[t_{a}, t_{b}\right]$ if $t_{a}<t_{b}$ or $t_{b}<t_{a}$ or $\left(t_{a}, t_{b}\right) \in T c$

Proof. On the one hand, the fact that $t_{a} \prec t_{b}$ or $t_{b} \prec t_{a}$ implies that there must be a cyclic subsequence including both $t_{a}$ and $t_{b}$, since they belong to a t-invariant (Property 1 ); thus since they have been observed consecutively there exists one place between them for assuring the consecutive firing. On the other hand, the $T c$ relation clearly states this dependency.

The set of transitions in a causal relation in $S$, is defined as: CausalR $=\left\{\left(t_{a}, t_{b}\right) \mid\left(t_{a}<t_{b} \wedge\left(t_{a} \prec t_{b} \vee t_{b} \prec t_{a}\right)\right) \vee\left(t_{a}, t_{b}\right) \in T c\right\}$.

From the sequence in Example 1, ConcR $=\left\{\left(t_{3}, t_{4}\right),\left(t_{4}, t_{3}\right)\right\}$ and CausalR $=\left\{\left(t_{1}, t_{2}\right),\left(t_{2}, t_{3}\right),\left(t_{4}, t_{1}\right),\left(t_{4}, t_{5}\right),\left(t_{5}, t_{6}\right),\left(t_{6}, t_{7}\right),\left(t_{7}, t_{4}\right)\right.$, $\left.\left(t_{2}, t_{4}\right),\left(t_{3}, t_{1}\right)\right\}$.

It is possible that several transition pairs in $S e q$ cannot be classed as causal or as concurrent, for example $\left(t_{3}, t_{5}\right)$. Such pairs, contained in $S e q^{\prime}=((\operatorname{Seq} \backslash$ CausalR $) \backslash$ ConcR, will be treated later.

Remark 1: The computational complexity of finding the previous relations is $\mathrm{O}(|S|)$ in the worst case, for the sequential and repetitive dependence relations, and $\mathrm{O}\left(|T|^{2}\right)$ for computing causal and concurrent relations.

\section{COMPUTING THE T-INVARIANTS}

Based on the previous definitions and properties, a technique for determining the t-invariants is proposed. A first approximation to the supports of the t-invariants is $R d\left(t_{j}\right)$ (Property 1). The main challenge is to discover the $\mathrm{t}$ invariants whose transitions appear interleaved in $S$. In particular, complex situations can appear when the transitions in a $R d\left(t_{j}\right)$ need for its execution the firing of other transitions not included in it; also when two or more t-invariants share transitions.

\section{A. Extending the repetitive dependencies}

In order to determine the t-invariants it is necessary to extend the $R d$ sets to obtain the supports of the invariants, by using additional notions introduced below.

Definition 9. A transition $t_{a}$ is indirect repetitive dependent of $t_{c}$ denoted as $t_{a} \prec \prec t_{c}$ iff there is a transition $t_{b}$ such that $\left(t_{a}\right.$ $\left.\prec t_{b}\right)$ and $\left(t_{b} \prec t_{c}\right)$. Therefore, the indirect repetitive dependent set is $\operatorname{IRd}\left(t_{a}\right)=\left\{t_{c} \mid t_{a}<<t_{c}\right\}$. The transitive extension of a $\operatorname{Rd}\left(t_{a}\right)$ is $R d_{e x}\left(t_{a}\right)=R d\left(t_{a}\right) \cup \operatorname{Ird}\left(t_{a}\right)$.

Property 2. All the transitions in a $R d_{e x}\left(t_{a}\right)$ belong to the support of a t-invariant.

Proof. It follows from Property 1 and Definition 7; if the firing of $t_{b}$ is conditioned to the firing of $t_{c}$, and $t_{a} \prec t_{b}$, then the firing of $t_{a}$ is also conditioned to the firing of all the $t_{c} \in R d\left(t_{b}\right)$, even if $t_{c}$ does not always appears between two occurrences of $t_{a}\left(t_{c} \notin R d\left(t_{a}\right)\right)$.

$R d_{e x}$ sets approximate the supports of t-invariants; thus it is necessary to enlarge these sets. For this purpose, relevant $R d_{e x}$ have to be handled.

Definition 10. A $R d_{e x}\left(t_{j}\right)$ set is said to be maximal iff there is no other $R d_{e x}\left(t_{k}\right)$ that includes $R d_{e x}\left(t_{j}\right) . R d M=\left\{R d M_{i} \mid R d M_{i}\right.$ is a maximal $\left.R d_{e x}\left(t_{j}\right)\right\}$.

Example 2. Consider the set of tasks $T=\left\{t_{0}, t_{1}, t_{2}, t_{3}, t_{4}, t_{5}\right.$, $\left.t_{6}, t_{7}\right\}$ and the sequence $S=t_{6} t_{1} t_{7} t_{4} t_{6} t_{1} t_{7} t_{4} t_{6} t_{1} t_{2} t_{4} t_{6} t_{0} t_{7} t_{3} t_{6} t_{0}$ $t_{2} t_{3} t_{6} t_{1} t_{7} t_{4} t_{6} t_{1} t_{7} t_{4} t_{6} t_{0} t_{2} t_{3} t_{6} t_{1} t_{2} t_{4} t_{6} t_{0} t_{2} t_{3} t_{6} t_{1} t_{2} t_{4} t_{6} t_{1} t_{2} t_{4} t_{6} t_{0} t_{2}$ $t_{3} t_{6} t_{0} t_{7} t_{3} t_{6} t_{0} t_{7} t_{5} t_{4} t_{6} t_{0} t_{7} t_{3} t_{6} t_{0} t_{5} t_{2} t_{4} t_{6} t_{0} t_{5} t_{7} t_{4} t_{6} t_{1} t_{7} t_{4} t_{6} t_{1} t_{2} t_{4} t_{6}$ $t_{0} t_{5} t_{2} t_{4} t_{6} t_{1} t_{7} t_{4} t_{6} t_{1} t_{2} t_{4} t_{6} t_{0} t_{2} t_{3} t_{6} t_{1} t_{2} t_{4} t_{6} t_{1} t_{2} t_{4} t_{6} t_{1} t_{2} t_{4} t_{6} t_{1} t_{2} t_{4} t_{6}$ $t_{0} t_{5} t_{7} t_{4} t_{6} t_{1} t_{2} t_{4} t_{6} t_{0} t_{7} t_{3} t_{6} t_{0} t_{5} t_{2} t_{4} t_{6} t_{0} t_{2} t_{5} t_{4} t_{6} t_{1} t_{7} t_{4} t_{6} t_{1} t_{7} t_{4} t_{6} t_{0} t_{7}$ $t_{3} t_{6} t_{1} t_{7} t_{4} t_{6} t_{0} t_{7} t_{5} t_{4} t_{6} t_{1} t_{2} t_{4} t_{6} t_{0} t_{5} t_{7} t_{4} t_{6} t_{0} t_{7} t_{3} t_{6} t_{0} t_{2} t_{5} t_{4} t_{6} t_{0} t_{5} t_{7} t_{4}$ $t_{6} t_{0} t_{2} t_{5} t_{4} t_{6} t_{1} t_{7} t_{4} t_{6} t_{0} t_{7} t_{3} t_{6} t_{0} t_{2} t_{5}, \ldots$, where $|S|=200$.

The repetitive dependencies computed from $S$ are: $R d\left(t_{0}\right)=$ $\left\{t_{0}, t_{6}\right\}, R d\left(t_{1}\right)=\left\{t_{1}, t_{6}, t_{4}\right\}, R d\left(t_{2}\right)=\left\{t_{2}, t_{6}\right\}, R d\left(t_{3}\right)=\left\{t_{3}, t_{0}\right.$, $\left.t_{6}\right\}, R d\left(t_{4}\right)=\left\{t_{4}, t_{6}\right\}, R d\left(t_{5}\right)=\left\{t_{5}, t_{6}, t_{4}, t_{0}\right\}, \operatorname{Rd}\left(t_{6}\right)=\left\{t_{6}\right\}$, $R d\left(t_{7}\right)=\left\{t_{7}, t_{6}\right\}$; the transitive extension does not modify these sets, i.e. $\operatorname{Ird}\left(t_{i}\right)=\operatorname{Rd}\left(t_{i}\right)$.

The computed $R d M_{i}$ are: $R d M_{1}=\left\{t_{5}, t_{6}, t_{4}, t_{0}\right\}, R d M_{2}=\left\{t_{1}\right.$, $\left.t_{6}, t_{4}\right\}, R d M_{3}=\left\{t_{3}, t_{6}, t_{0}\right\}, R d M_{4}=\left\{t_{7}, t_{6}\right\}, R d M_{5}=\left\{t_{2}, t_{6}\right\}$. Other relations deduced from $S$ are summarised in Table 1. 


\begin{tabular}{|c|c|c|c|c|c|}
\hline$T_{i}$ & $\begin{array}{c}\text { Seq } \\
\left(\bullet<t_{j}\right)\end{array}$ & $\begin{array}{c}\text { CausalR } \\
\left(\bullet, t_{j}\right)\end{array}$ & $\begin{array}{c}\text { ConcR } \\
\left(\bullet \| t_{j}\right)\end{array}$ & $\begin{array}{c}\text { Seq } \\
\left(\bullet<t_{j}\right)\end{array}$ & $\begin{array}{c}T \times T S e q \\
\left(\bullet><t_{j}\right)\end{array}$ \\
\hline$t_{0}$ & $t_{2}, t_{5}, t_{7}$ & $t_{5}$ & & $t_{2}, t_{7}$ & $t_{1}, t_{3}, t_{4} t_{6}$ \\
\hline$t_{1}$ & $t_{2}, t_{7}$ & & & $t_{2}, t_{7}$ & $t_{0}, t_{1}, t_{3}, t_{4}, t_{5}, t_{6}$ \\
\hline$t_{2}$ & $t_{3}, t_{5}, t_{4}$ & & $t_{5}$ & $t_{3}, t_{4}$ & $t_{1}, t_{0}, t_{7}, t_{6}$ \\
\hline$t_{3}$ & $t_{6}$ & $t_{6}$ & & & $t_{0}, t_{1}, t_{2}, t_{4}, t_{5}, t_{7}$ \\
\hline$t_{4}$ & $t_{6}$ & $t_{6}$ & & & $t_{0}, t_{1}, t_{2}, t_{3}, t_{5}, t_{7}$ \\
\hline$t_{5}$ & $t_{2}, t_{4}, t_{7}$ & $t_{4}$ & $t_{2}, t_{7}$ & & $t_{1}, t_{0}, t_{3}, t_{4} t_{6}$ \\
\hline$t_{6}$ & $t_{0}, t_{1}$ & $t_{1}, t_{0}$ & & & $t_{7}, t_{2}, t_{3}, t_{4} t_{5}$ \\
\hline$t_{7}$ & $t_{3}, t_{5}, t_{4}$ & & $t_{5}$ & $t_{3}, t_{4}$ & $t_{1}, t_{0}, t_{2}, t_{6}$ \\
\hline
\end{tabular}

Table1. Relations between tasks in Example 2.

The knowledge of transitions that belong only to one $R d M_{i}$ will be useful for determining the invariants.

Definition 11. The set of transitions that belong to only one $R d M_{i}$ is $T_{R d M}=\bigcup_{i=1}^{r}\left(R d M_{i} \backslash \bigcup_{\mathrm{j}=1, \mathrm{j} \neq \mathrm{i}}^{\mathrm{r}} R d M_{j}\right)$ where $\mathrm{r}=|R d M|$.

Now it is possible to enlarge these sets by merging $R d M_{i}$ that share common transitions. This can be done when the $R d M_{i}$ fulfils several conditions stated below.

Proposition 3. All the transitions in a $R d M_{x, y}=R d M_{x} \cup R d M_{y}$ are included in the support of a $\mathrm{t}-$ invariant if there exist $t_{i} \in R d M_{x}$ and $t_{j} \in R d M_{y}$ such that i) $\left(t_{i}, t_{j}\right) \in \operatorname{ConcR}$, and ii) $R d\left(t_{i}\right) \cap R d\left(t_{j}\right) \neq \varnothing$.

Proof. Let be $t_{k} \in R d\left(t_{i}\right) \cap R d\left(t_{j}\right)$. Since $\left(t_{i}, t_{j}\right) \in \operatorname{ConcR}$, the subsequence $t_{i} t_{j} \ldots t_{k} \ldots t_{j} t_{i} \ldots t_{k} \ldots t_{i} t_{j} \ldots t_{k}$ is found in $S$; that is, both transitions $t_{i}$ and $t_{j}$ appear between the occurrences of $\mathrm{t}_{\mathrm{k}}$. Therefore $t_{i}$ and $t_{j}$ belong to a largest repetitive dependence $R d M_{x, y}=R d M_{x} \cup R d M_{y}$, which is part of the support of a $\mathrm{t}$ invariant. $\square$

The next procedure obtains $R d M^{+}$, the set of extensions of $R d M_{i}$ by performing the union operation between members of $R d M$.

Algorithm 1. Merging RdMs

Input: $R d M=\left\{R d M_{1}, R d M_{2} \ldots R d M_{r}\right\}$

Output: $R d M^{+}$

1. $R d M^{+} \leftarrow R d M$

2. $\forall\left(t_{i}, t_{j}\right) \in$ ConcR

$$
\begin{aligned}
\text { If } R d\left(t_{i}\right) & \cap R d\left(t_{j}\right) \neq \varnothing \text { then } \\
& R d M_{x, y} \leftarrow R d M_{x} \cup R d M_{y} \\
& R d M^{+} \leftarrow R d M^{+} \cup\left\{R d M_{x, y}\right\}
\end{aligned}
$$

After applying this procedure to $R d M$ obtained in Example 2 , given that $\left(t_{5} \| t_{7}\right), \quad\left(t_{2} \| t_{5}\right)$, and $R d\left(t_{5}\right) \cap R d\left(t_{7}\right) \neq \varnothing$ and $R d\left(t_{5}\right) \cap R d\left(t_{2}\right) \neq \varnothing$, two new maximal sets are obtained: $R d M_{l, 4}$ $=R d M_{1} \cup R d M_{4}, R d M_{1,5}=R d M_{l} \cup R d M_{5}$. Then $R d M^{+}=\left\{R d M_{1}\right.$, $\left.R d M_{2}, R d M_{3}, R d M_{4}, R d M_{5}, R d M_{1,4}, R d M_{1,5}\right\}$.

\section{B. Finding the repetitive behaviour}

A t-invariant induces a sub-graph of the $P N$ model, called repetitive component or t-component. In the case of a deadlock-free and 1-bounded $P N$ the t-component is strongly connected $(S c)$. We will analyse the extended $R M S_{i}$ through a graph representation of CausalR and the transition pairs in Seq'.
Definition 12. The Graph of causality relations between tasks, named causality graph of a $R d M_{i}$, is a digraph denoted $G_{i}$, defined as follows.

$$
\begin{aligned}
& G_{i}=\left(V_{i}, E_{i}\right) ; V_{i}=\left\{t_{k} \mid t_{k} \in R_{d} M_{i}\right\} \\
& E_{i}=\left\{\left(t_{k}, t_{l}\right) \in V_{i} \times V_{i} \mid\left(t_{k}, t_{l}\right) \in \text { CausalR } \bigcup S e q^{\prime}\right\}
\end{aligned}
$$

The set of causality graphs corresponding to $R d M^{+}$is denoted $C G=\left\{G_{l}, G_{2} \ldots G_{q}\right\}$, where $G_{i}$ is the causality graph of a $R d M_{i}$. A $G_{i}$ is maximal iff there is not a $G_{k} \in C G$ such that $G_{i} \subset G_{k}$.

The set $C G$ corresponding to the $R d M^{+}$computed before for Example 2 is shown in Figure 1.

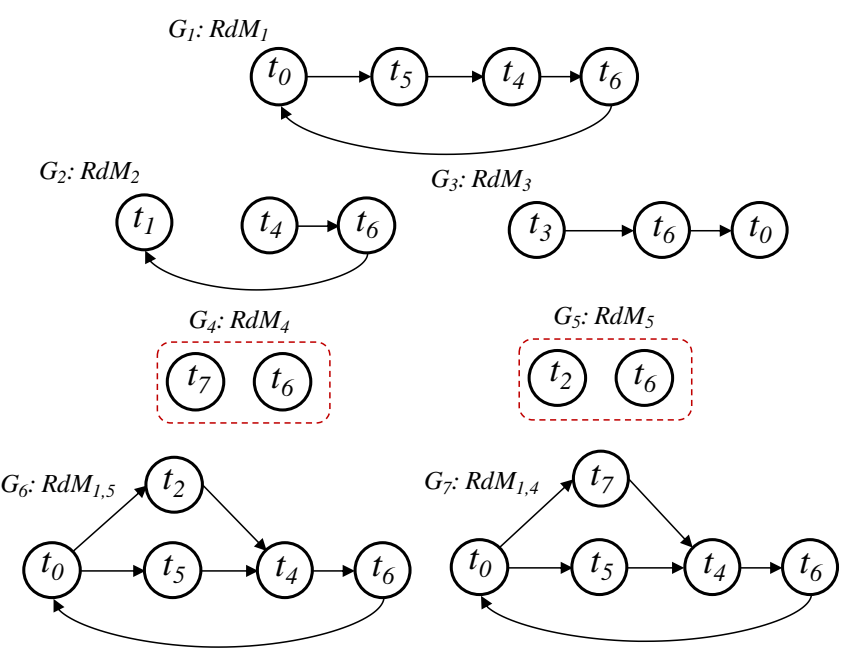

Figure 1. $C G$ corresponding to $R D M^{+}$of Example 2.

Theorem 1. Let $G_{i}$ be a causality graph in $C G$. If a maximal $G_{i}$ is $S c$, then its nodes are the support of some minimum t-invariant of the $P N$ that reproduces $S$.

Proof. The vertices of $G_{i}$ correspond to a $R d M_{i}$ whose transitions are included in the support of a t-invariant $\mathrm{Y}_{\mathrm{i}}$ (Proposition 3). Suppose that the transitions in $V_{i}$ are not the support of a t-invariant; then there exists at least a $t_{k} \notin V_{i}$ such that $t_{k} \in\left\langle Y_{i}\right\rangle$ that must fire to allow the repetitive firing of transitions in $V_{i}$ together with $t_{k}$; thus there are not cycles containing $t_{k}$ in $G_{i}$, consequently it is not $S c$.

If the connectivity test is applied to the graphs in $C G$, it may occur that some $G_{i}$ are not $S c$. Then it is possible to obtain larger graphs by merging $G_{i}$ with common vertices, through a merging operation of graphs defined below.

Definition 13. The merging operation $\left(\cup_{G}\right)$ of two causality graphs $G_{i} \cup_{G} G_{j}$ produces a new graph $G_{i, j}$.

$$
\begin{aligned}
& G_{i, j}=\left(V_{i, j}, E\right) ; V_{i, j}=V_{i} \cup V_{j} \\
& E=\left\{\left(t_{k}, t_{l}\right) \in V_{i, j} \times V_{i, j} \mid\left(t_{k}, t_{l}\right) \in \text { CausalR } \bigcup S e q^{\prime}\right\}
\end{aligned}
$$

Figure 2 shows the merging of the graphs $G_{2}$ and $G_{5}$. The idea is to merge iteratively graphs $G_{i}, G_{j} \in G C$ such that $V_{i}$ $\bigcap V_{j} \neq \varnothing$. In each iteration every $G_{i, j}$ produced must not include other $S c$ graphs. Based on this strategy, a procedure for computing all the $S c$ graphs from CG is presented below. 


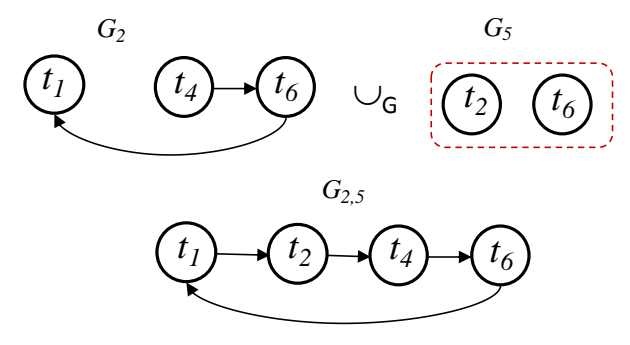

Figure 2. $G_{2} \cup_{\mathrm{G}} G_{5}$, where $\left(t_{1}, t_{2}\right),\left(t_{2}, t_{4}\right) \in$ CausalR $\cup$ Seq

Algorithm 2. Getting the t-invariants from $S$

Input: $C G=\left\{G_{l}, G_{2} \ldots G_{q}\right\}$

Output: $\langle\mathrm{Y}(\mathrm{S})\rangle$ : Supports of t-invariants

1. $G_{S c} \leftarrow$ all maximal $S c G_{i} \in C G$

2. $G_{N S c} \leftarrow$ all non $S c G_{i} \in C G$

3. $l_{N S c} \leftarrow\left|G_{N S c}\right|$

4. For 1 to $l_{N S c}$

$$
\begin{aligned}
& \text { 4.1 } \forall G_{i} \in G_{N S c} \\
& \forall G_{j} \in G_{N S c} \mid G_{i} \neq G_{j} \text { and } G_{i} \cap G_{j} \neq \varnothing \\
& \text { a) } G_{i, j} \leftarrow G_{i} \cup_{\mathrm{G}} G_{j} \\
& \text { b) If } G_{i, j} \text { is Sc and } \forall G_{k} \in G_{S c}, G_{k} \not \subset G_{i, j} \\
& \text { then } G_{S c} \leftarrow G_{S c} \cup G_{i, j} \\
& \text { else NewNSc } \leftarrow N e w N S c \cup\left\{G_{i, j}\right\} \\
& 4.2 G_{N S c} \leftarrow N e w N S c ; N e w N S c \leftarrow \varnothing \\
& \text { 5. Return } G_{S c}
\end{aligned}
$$

The above algorithm ensures that the nodes of each $G_{i} \in$ $G_{S c}$ correspond to the support of minimal t-invariants.

Remark 2. The computational complexity of finding the supports of T-invariants when no $G_{i} \in C G$ is strongly connected (worst case) is $O\left(\left|G_{N S c}\right|^{3}\right)$. However, the worst case is unlikely since when $G_{i, j}$ is built (step 4.1.a), the $G_{i}$ that are $S c$ are discarded. Furthermore if $G_{i, j}$ is $S c$ but if it contains other $G_{k}$ that is $S c$ (step 4.1.b), then $G_{i, j}$ is also discarded.

Theorem 2. Algorithm 2 obtains all the supports of the minimal t-invariants of a $P N$ model that reproduces the task sequence $S$.

Proof. This procedure performs exhaustively the union of graphs which are not $S c$ and have common vertices. In every iteration, the formed $S c$ graphs are no longer considered in the union operations; this reduces progressively the number of non $S c$ graphs. Since it is avoided using the already obtained $S c$ graphs; this guarantees finding minimal Sc graphs and then the support of minimal invariants. When it is not possible to generate new $S c$ graphs the procedure stops. Every $V_{i}$ of $G_{i}$ in $G_{s c}$ is the support of a t-invariant.

The set of obtained t-invariants is $\mathrm{Y}(\mathrm{S})=\left\{\mathrm{Y}_{\mathrm{i}} \mid \mathrm{Y}_{\mathrm{i}}\right.$ is the vector corresponding to $V_{i}$ \}

When Algorithm 2 is applied to $C G$ of Example 2, the resulting supports of t-invariants are $\left\langle\mathrm{Y}_{1}\right\rangle=\left\{t_{0}, t_{4}, t_{5}, t_{6}, t_{7}\right\}$, $\left\langle\mathrm{Y}_{2}\right\rangle=\left\{t_{0}, t_{4}, t_{5}, t_{6}, t_{2}\right\}, \quad\left\langle\mathrm{Y}_{3}\right\rangle=\left\{t_{1}, t_{4}, t_{6}, t_{2}\right\}, \quad\left\langle\mathrm{Y}_{4}\right\rangle=\left\{t_{1}, t_{4}, t_{6}\right.$, $\left.t_{7}\right\},\left\langle\mathrm{Y}_{5}\right\rangle=\left\{t_{0}, t_{3}, t_{6}, t_{2}\right\}$, and $\left\langle\mathrm{Y}_{6}\right\rangle=\left\{t_{0}, t_{3}, t_{6}, t_{7}\right\}$.

\section{BUILDING THE PN MODEL}

Causal relations $\left[t_{i}, t_{j}\right]$ determine the existence of a place between transitions. Using this basic structure, named dependency, and the knowledge of t-invariants, a technique for building a PN model is now presented.

\section{A. Merging transitions of dependencies}

All the transitions named $t_{i}$ within several dependencies must be merged into a single one.

Rule 1. Two dependencies in the form $\left[t_{i}, t_{j}\right]$ and $\left[t_{j}, t_{k}\right]$ produce, straightforward, a sequential sub-structure including two places, which allows the firing of the sequence $t_{i} t_{j} t_{k}$, as illustrated in Fig 3.a).

Rule 2. When the first transitions in two dependencies are the same $\left(\left[t_{i}, t_{j}\right]\right.$ and $\left.\left[t_{i}, t_{k}\right]\right)$, two possible substructures can be created (Fig. 3.b):

a) The places of the dependencies are merged into a single one iff $t_{j}$ and $t_{k}$ belong to different t-invariants. This is denoted as $\left[t_{i}, t_{j}+t_{k}\right]$. This rule applies most of the time, but a special situation could appear when $t_{j} \mid t_{k}$; in this case the dependency $\left[t_{i}, t_{j}+t_{k}\right]$ is not created.

b) The places of the dependencies are not merged iff $t_{j}$ and $t_{k}$ belong to a same t-invariant. This is denoted as $\left[t_{i}, t_{j}|| t_{k}\right]$.

Similarly, for dependencies having a common second transition $\left(\left[t_{i}, t_{k}\right]\right.$ and $\left.\left[t_{j}, t_{k}\right]\right)$, the substructure created will be either $\left[t_{i}+t_{j}, t_{k}\right]$ or $\left[t_{i} \| t_{j}, t_{k}\right]$ (Fig. 3.b). In both cases the observations $\left(t_{i}, t_{j}\right), \quad\left(t_{i}, t_{k}\right), \quad\left(t_{j}, t_{k}\right) \in S e q$, deriving the dependencies, are preserved. This merging rule is illustrated in Figure 3. In general, a set of dependencies in the form $\left\{\left[t_{i}, t_{j}\right]\right.$, $\left.\left[t_{i}, t_{k}\right], \ldots\left[t_{i}, t_{r}\right]\right\}$ may produce either $\left[t_{i}, t_{j}+t_{k}+\ldots+t_{r}\right]$ or $\left[t_{i}\right.$, $\left.t_{j}\left\|t_{k}\right\| \ldots \| t_{r}\right]$ according to the relations between transitions $t_{j}$, $t_{k}, \ldots, t_{r}$.
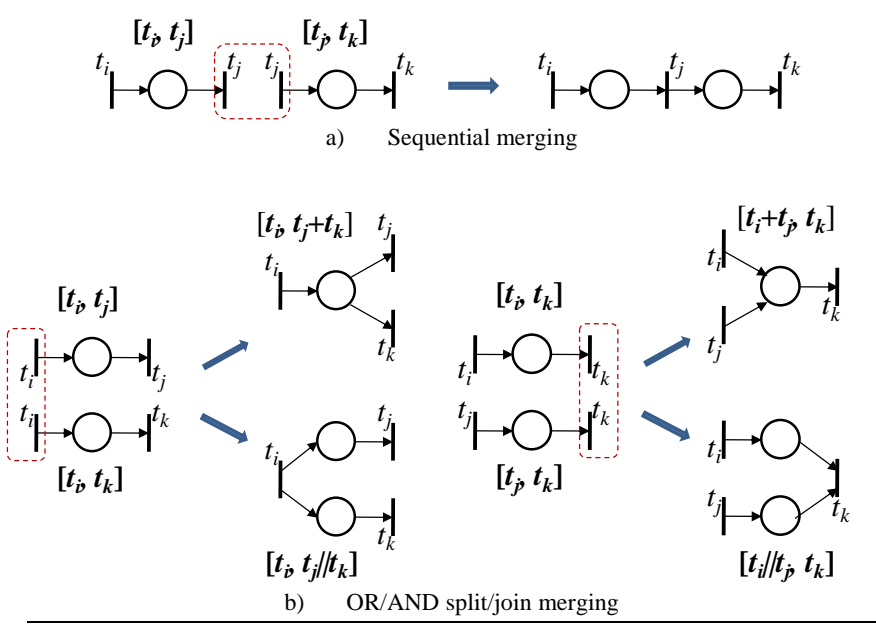

Figure 3. Rules for merging dependencies

Consequently, the merging can be applied to composed dependencies that coincide with one expression of transitions of type $t_{i}+t_{j}$ or $t_{i}|| t_{j}$; for example $\left[t_{i}+t_{j}, t_{k}\right]$ and $\left[t_{i}+t_{j}, t_{r}\right]$ leads to $\left[t_{i}+t_{j}, t_{k}+t_{r}\right]$ if both $t_{k}$ and $t_{r}$ do not belong to the same invariant. 
The application of these merging rules to the dependencies derived from the pairs in CausalR $\cup S e q$ ', leads to a PN model $N_{l}$ including all the transitions.

In Example 2, the application of rules 1 and 2 to the obtained relations in CausalR $\cup S e q$ ' of Table 1 yields the set of composed dependencies: $\left[t_{5}, t_{4}\right],\left[t_{0}, t_{2} \| t_{5}\right],\left[t_{0}, t_{5} \| t_{7}\right],\left[t_{0}\right.$, $\left.t_{2}+t_{7}\right],\left[t_{1}, t_{2}+t_{7}\right],\left[t_{2}, t_{3}+t_{4}\right],\left[t_{7}, t_{3}+t_{4}\right],\left[t_{4}+t_{3}, t_{6}\right],\left[t_{6}, t_{0}+t_{1}\right]$, $\left[t_{0}+t_{1}, t_{2}\right],\left[t_{0}+t_{1}, t_{7}\right],\left[t_{2} \| t_{5}, t_{4}\right],\left[t_{7} \| t_{5}, t_{4}\right],\left[t_{7}+t_{2}, t_{3}\right]\left[t_{7}+t_{2}, t_{4}\right]$. Afterwards the obtained dependencies are $\boldsymbol{p}_{0}:\left[t_{6}, t_{1}+t_{0}\right]$, $\boldsymbol{p}_{1}:\left[t_{0}+t_{1}, t_{2}+t_{7}\right], \boldsymbol{p}_{1}, \boldsymbol{p}_{2}:\left[t_{0},\left(t_{2}+t_{7}\right) \| t_{5}\right], \boldsymbol{p}_{\mathbf{3}}:\left[t_{5}, t_{4}\right], \quad \boldsymbol{p}_{4}:\left[t_{2}+t_{7}\right.$, $\left.t_{4}+t_{3}\right], \boldsymbol{p}_{5}:\left[t_{4}+t_{3}, t_{6}\right]$. The sequential merging of substructures of the dependencies yields the $P N$ model $N_{l}$ shown in Figure 4.

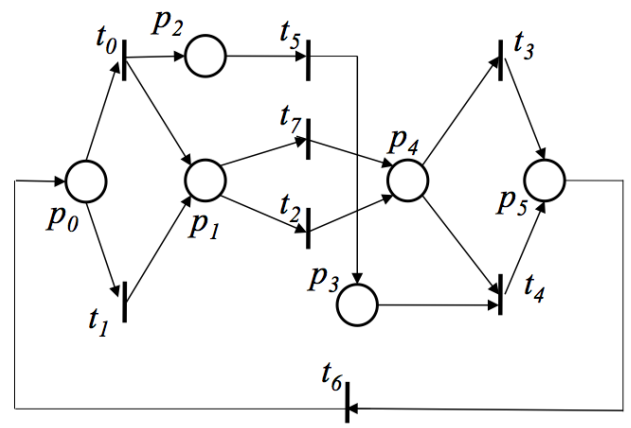

Figure 4. $N_{I}$ built from $S$ of example 2.

\section{B. Model adjustment}

Although $S$ may be fired in $N_{l}$ most of the times, the obtained model could not fire $S$, or could fire $S$ but also exceeding sequences. The $P N$ in Figure 4 does not reproduce $S$ of Example 2 in particular the subsequences $t_{1} t_{2} t_{4}$ and $t_{1} t_{7} t_{4}$ cannot be fired in $N_{l}$. This is because the computed tinvariants $\mathrm{Y}(S)$ differ from those of $N_{l}\left(\mathrm{~J}\left(N_{l}\right)\right)$. If $\mathrm{Y}(S)$ coincide with $\mathrm{J}\left(N_{l}\right)$, then $N_{l}$ is the correct model; otherwise it must be adjusted.

The mismatching between $\mathrm{Y}(S)$ and $\mathrm{J}\left(N_{l}\right)$ is due to the fact that the computed model does not include $P N$ elements (places and arcs) which assure implicit behaviours not exhibited in $S$, named implicit dependencies.

Definition 14. In a 1-bounded PN, $\left[t_{i}, t_{j}\right]$ is called an implicit dependency, if although there is a place between the transitions, the firing of $t_{i}$ does not produce a marking that enables $t_{j}$. It is necessary the firing of at least one transition before $t_{j}$.

In a $P N$ model, implicit dependencies represent the record of the occurrence of a $t_{i}$, which is used as condition to enable a future event $t_{j}$. In general, an implicit dependency represents a constraint in the flow of tokens in the net by assuring that $t_{j}$ is fired only when $t_{i}$ is fired before; otherwise the absence of such a dependency will allow the firing of exceeding sequences in the remainder model.

When $\mathrm{Y}(S) \neq \mathrm{J}\left(N_{l}\right), \quad N_{l}$ must therefore be adjusted by finding the pertinent implicit dependencies that extend $N_{l}$ into $N_{2}$, whose t-invariants agree with $\mathrm{Y}(S)$. In order to amend $N_{l}$, two cases of mismatching are considered: 1) $\mathrm{Y}(S) \subset \mathrm{J}\left(N_{1}\right)$, or 2) $\mathrm{Y}(S) \neq \mathrm{J}\left(N_{l}\right)$ and $\mathrm{Y}(S) \not \subset \mathrm{J}\left(N_{l}\right)$, i.e. $\exists \mathrm{Y}_{\mathrm{i}} \in \mathrm{Y}(S)$ such that $\mathrm{Y}_{\mathrm{i}} \notin \mathrm{J}\left(N_{l}\right)$. The handling of each case is described below.
Case 1

In this case $N_{l}$ has more invariants than those computed from $\mathrm{S}$; thus it represents an exceeding behaviour. A new place between two transitions $t_{i}$ and $t_{j}$ has to be added to $N_{l}$ in order to constrain the differed firing of $t_{j}$ after the firing of $t_{i}$.

Proposition 4. A dependency $\left[t_{i}, t_{j}\right]$ must be added to $N_{l}$ if the following condition holds: $\left(t_{i}\right\rangle\left\langle t_{j}\right) \wedge\left(t_{i}, t_{j} \in\left\langle\mathrm{Y}_{\mathrm{k}}\right\rangle\right) \wedge\left(t_{i}\right.$, $\left.t_{j} \in T_{R D M}\right)$

Proof. If $\left[t_{i}, t_{j}\right]$ must not be added, it is because i) $t_{i}$ and $t_{j}$ have been observed consecutively $\left(t_{i}<t_{j}\right)$, or ii) each transition belongs to a different t-component, or iii) at least one of $t_{i}, t_{j}$ does not belong to a $T_{R D M}$.

Case 2

Let $\mathbf{J}\left(N_{l}\right)=\left\{\mathrm{J}_{1}, \mathrm{~J}_{2}, . ., \mathrm{J}_{\mathrm{r}}\right\}$ be the set of t-invariants of $N_{l}$, such that $\mathrm{CJ}_{\mathrm{j}}=0$, where $\mathrm{C}$ is the incidence matrix of $N_{l}$. Consider a $\mathrm{Y}_{\mathrm{r}} \notin \mathrm{J}\left(N_{l}\right)$. Let $p_{k}$ be the place corresponding to the row in which $C Y_{\mathrm{r}} \neq 0$ (i.e. $\mathrm{C}\left(p_{k}\right) \mathrm{Y}_{\mathrm{r}} \neq 0$ ). In order to obtain the dependency $\left[t_{i}, t_{j}\right]$, other transition in $N_{l}$ must be linked through $p_{k}$ to one of the transitions in ${ }^{\bullet} p_{k}$ or $p_{k} \bullet$ according to the following rule.

Proposition 5. A dependency $\left[t_{i}, t_{j}\right]$ must be added to $N_{l}$ if $\left.t_{i}\right\rangle\left\langle t_{j}\right.$, and $t_{i}, t_{j} \in\left\langle\mathrm{Y}_{\mathrm{r}}\right\rangle$, and if one of the following conditions holds: i) $t_{i} \in p_{k}$ and $t_{j} \in T_{R D M}$, when $\left|\bullet p_{k}\right|<\mid p_{k} \bullet$, or ii) $t_{j} \in p_{k}^{\bullet}$ and $t_{i} \in T_{R D M}$, when $\left|\bullet p_{k}\right|>\mid p_{k} \bullet$. This dependency ensures that $\mathrm{C}\left(p_{k}\right) \mathrm{Y}_{\mathrm{r}}=0$.

Proof. The two first conditions are the same than those of Case 1 . We will analyse the conditions regarding $\bullet p_{k}$ and $p_{k} \bullet$. In both situations $\left|\bullet p_{k}\right|$ and $\left|p_{k} \bullet\right|$ are unbalanced and one of $t_{i}$ or $t_{j}$ has to be related to one of $\bullet p_{k}$ and $p_{k} \bullet$ accordingly, to enforce $\mathrm{J}_{\mathrm{r}}$ as t-invariant of $N_{l}$. Furthermore $\left|\bullet p_{k}\right|=\mid p_{k} \bullet$ yielding $\mathrm{C}\left(p_{k}\right) \mathrm{Y}_{\mathrm{r}}=0$.

When all the corrections to $N_{l}$ are done, it is possible that $\mathrm{Y}(S) \subset \mathrm{J}\left(N_{1}\right)$, then the rule of Case 1 is applied and the new model $N_{2}$ fulfils $\mathrm{Y}(S)=\mathrm{J}\left(N_{2}\right)$. Algorithm 3 summarises the procedure to obtain the implicit dependencies.

Algorithm 3. Finding implicit dependencies

Input: $N_{l}, \mathrm{~J}\left(N_{l}\right), \mathrm{Y}(S)$

Output: $N_{2}$

1. If $\mathrm{Y}(S) \subset \mathrm{J}\left(N_{l}\right)$

a) $\forall\left(t_{i}, t_{j}\right) \mid t_{i},><t_{j} \wedge t_{i}, t_{j} \in T_{R d M} \wedge t_{i}, t_{j} \in \mathrm{y}_{\mathrm{i}}$ add a place between $\left(t_{i}, t_{j}\right)$

2. If $\exists \mathrm{y}_{\mathrm{i}} \in \mathrm{Y}(S) \mid \mathrm{y}_{\mathrm{i}} \notin \mathrm{J}\left(N_{l}\right)$

a) Find a $p_{k} \mid \mathrm{C}\left(p_{k}\right) \mathrm{y}_{\mathrm{i}} \neq 0$

b) Add $\left[t_{i}, t_{j}\right]$ through $p_{k}$ relations that fulfil

$$
\begin{aligned}
& t_{i},><t_{j} \wedge t_{i}, t_{j} \in \mathrm{y}_{\mathrm{i}} \wedge\left(t_{i} \in \bullet p_{k}, t_{j} \in T_{R D M}\right) \text { or } \\
& t_{i},><t_{j} \wedge t_{i}, t_{j} \in \mathrm{y}_{\mathrm{i}} \wedge\left(t_{j} \in p_{k}^{\bullet}, t_{i} \in T_{R D M}\right)
\end{aligned}
$$

Remark 3. The complexity of computing the implicit dependencies is $\mathrm{O}(|P| \times|T|)$; it is related to the matrix-vector product operation $\mathrm{C}\left(p_{k}\right) \mathrm{y}_{\mathrm{i}}$.

Let us analyze $N_{l}$ in Figure 4, obtained from $S$ in Example 2. First it is computed $\mathrm{J}\left(N_{l}\right)=\left\{\left\langle\mathrm{J}_{1}\right\rangle,\left\langle\mathrm{J}_{2}\right\rangle,\left\langle\mathrm{J}_{3}\right\rangle,\left\langle\mathrm{J}_{4}\right\rangle\right\} ;\left\langle\mathrm{J}_{1}\right\rangle=$ 
$\left\{t_{0}, t_{4}, t_{5}, t_{6}, t_{7}\right\},\left\langle\mathrm{J}_{2}\right\rangle=\left\{t_{0}, t_{4}, t_{5}, t_{6}, t_{2}\right\},\left\langle\mathrm{J}_{3}\right\rangle=\left\{t_{1}, t_{2}, t_{3}, t_{6}\right\}$, $\left\langle\mathrm{J}_{4}\right\rangle=\left\{t_{1}, t_{7}, t_{3}, t_{6}\right\}$. There is a mismatch between both sets and since $\mathrm{Y}(S) \not \subset \mathrm{J}\left(N_{l}\right)$, the problem is handled as in Case 2. It can be noticed that $\mathrm{Y}_{3}, \mathrm{Y}_{4}, \mathrm{Y}_{5}, \mathrm{Y}_{6} \notin \mathrm{J}\left(\mathrm{N}_{1}\right)$. In the analysis of $\mathrm{Y}_{3}, p_{k}=p_{3}$ because it fulfils the condition $C_{N I}\left(p_{3}\right) \mathrm{Y}_{\mathrm{i}} \neq 0$, as show in the next equation.

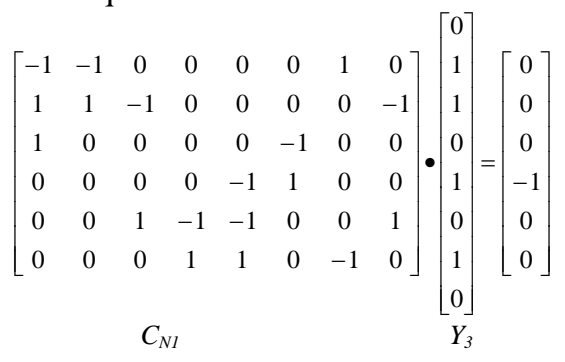

The transition in $t_{4} \in p_{k} \bullet$ is chosen to find the implicit dependency $\left[t_{i}, t_{4}\right]$. The transition that fulfils the conditions $\left.t_{i}\right\rangle\left\langle t_{4}, t_{i}, t_{4} \in\left\langle\mathrm{Y}_{3}\right\rangle, t_{i} \in T_{R d M}\right.$, is $t_{1}$; therefore the implicit dependency $\left[t_{l}, t_{4}\right]$ is added to $N_{l}$ by the corresponding arc $\left(t_{l}\right.$, $p_{3}$ ). Similarly $\mathrm{Y}_{4}, \mathrm{Y}_{5}, \mathrm{Y}_{6}$ are treated and the implicit dependency $\left[t_{0}, t_{3}\right]$ in $p_{2}$ is found. Finally the resulting PN model $N_{2}$, which exactly reproduces $S$ is shown in Figure 5 .

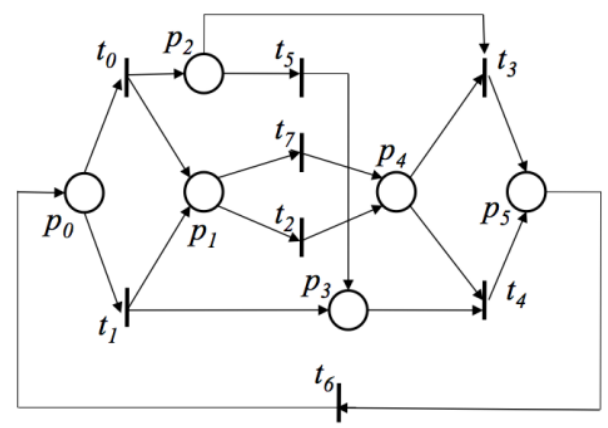

Figure 5. Resulting $P N N_{2}$ after model adjustment.

Theorem 3. Given a sequence of transitions $S \in T^{*}$, a 1bounded $P N$ model $N_{2}$ that reproduces $S$ can be obtained by applying the rules 1 and 2, and performing the adjustments of Algorithm 3.

Proof. Causality between transitions, established by the pairs in CausalR $\cup \mathrm{Seq}$ ' represents the precedence relationship between consecutive transitions in $S$ that are not in ConcR. The substructure associated to a dependency $\left[t_{i}, t_{j}\right]$ guarantees the consecutive firing of these transitions; thus by applying Rule 1 the flow expressed in CausalR $\cup$ Seq' is fulfilled by $N_{l}$. Furthermore, Rule 2 determines, by the knowledge of the tinvariants, whether the flow is split or joint in choice or parallel structures. Dependencies involving transitions included in $S c$ causality graphs assure the construction of repetitive components in $N_{l}$. Furthermore, adjustments to $N_{l}$ provided by Propositions 4 and 5 allow fitting the invariants computed form the observed behaviour with those of the discovered model.

\section{Initial marking}

The Initial marking must enable $S$; thus the procedure for determining $M_{0}$ is simple; it suffices a) to place tokens in the input places of the first transition in $S$, and b) executing the remainder $t_{j}$ in $\mathrm{S}$ and eventually adding tokens in some places of $\bullet t_{j}$ when the reached marking is not enough for firing $t_{j}$. In the case of example 2 , the only place initially marked in the PN Fig. 5 is $p_{5}$.

\section{Processing several event sequences}

This synthesis method may process $r$ event traces $S_{i}$ corresponding to the observed behaviour of the same discrete event process. The only constraint is that all the sequences must be sampled from the starting of the process. All the observed precedence relationships in $S e q_{i}$ of every $S_{i}$ are gathered into the $S e q$ relation at the beginning of the discovery procedure. The initial marking is determined for enabling every $S_{i}$.

\section{IMPLEMENTATION AND TESTS}

Algorithms derived from the proposed method have been implemented as a software tool and tested on numerous examples of diverse complexity. The tests were performed using the following scheme: first, a PN model is designed, and with the help of the PN editor/simulator PIPE [21], a long sequence $S$ is produced. Then the tool processes $S$ and the obtained model, coded in XML, is displayed using PIPE again.

Below we provide an example regarding a less simple PN model that can be discovered using the proposed PN discovery method. The model in Figure 6 has been obtained by processing the task $\log S=$ T16 T14 T2 T4 T3 T5 T9 T7 T3 T5 T9 T3 T5 T8 T17 T2 T3 T5 T9 T3 T4 T7 T5 T8 T11 T13 T15 T16 T1 T2 T4 T3 T5 T8 T6 T10 T17 T2 T3 T4 T5 T6 T9 T3 T5 T10 T9 T3 T5 T9 T3 T5 T9 T3 T5 T9 T3 T5 T9 T3 T5 T9 T3 T5 T9 T3 T5 T8 T17 T2 T4 T3 T7 T5 T9 T3 T5 T9 T3 T5 T8 T11 T12 T15 T16 T1 T2 T4 T7 T3 T5 T8 T11 T12 T15 T16 T14 T2 T3 T5 T8 T4 T6 T10 T17 T2 T3 T4 T6 T10 T5 T9 T3 T5 T8 T17 T2 T4 T6 T3 T10 T5 T9 T3 T5 T8 T17 T2 T3 T5 T8 T4 T6 T10 T11 T13 T15 T16 T14 T2 T4 T7 T3 T5 T9 T3 T5 T8 T11 T13 T15 T16 T1 T2 T3 T5 T9 T4 T6 T3 T10 T5 T9 T3 T5 T9 T3 T5 T9 T3 T5 T8 T17 T2 T3 T5 T9 T4 T3 T6 T5 T9 T3 T10 T5 T8 T11 T12 T15 T16 T14 T2 .., where $|S|=1500$.

This model includes diverse structures (nested tcomponent evolving concurrently) which are more complex than others published in literature. As a sign of performance, the processing time for $S$ in a laptop computer $(2.4 \mathrm{GHz}$ dualcore, Intel Core i5 processor, $4 \mathrm{~GB}$ of $1333 \mathrm{MHz}$ DDR3 memory) was about $3.6 \mathrm{~s}$.

Thanks to the software tool we developed it has been possible to test models of diverse structures, which include cycles nested into t-components, concurrency, and implicit dependencies. Special models such as two independent PNs concurrently evolving, and concurrent components related by mailbox places (message exchange) have been successfully built. This reveals the power of the method for dealing with black-box model discovery.

\section{CONCLUSION}

The proposed method for PN discovery handles long sequences $S_{i}$ representing the observed behaviour of a process from their initial states. No a priori knowledge about the number of places nor the start and end of tasks in traces $\sigma_{j}$ in $S_{i}$ is required. 


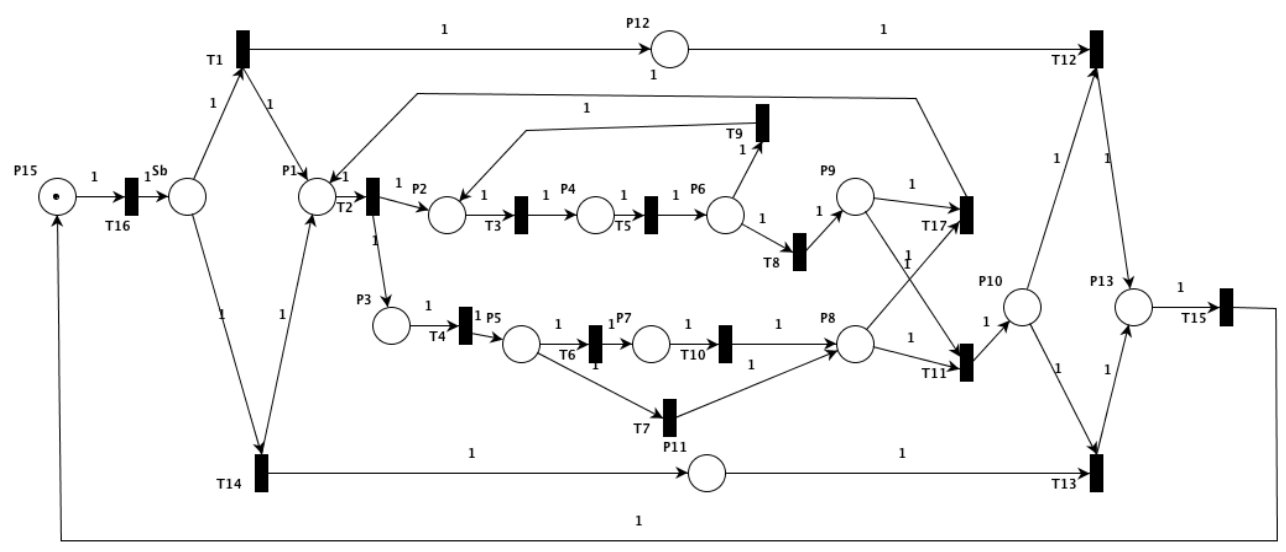

Figure 6. A non trivial discovered $\mathrm{PN}$ model from $\mathrm{S}_{3}$

This approach allows addressing efficiently discrete event processes exhibiting more complex behaviours than the approaches proposed in the fields of identification and process mining. This method is based mainly on searching the supports of t-invariants from the observed sequences $S_{i}$, and allows building an initial model which is adjusted later with the help of the computed t-invariants; the final model includes implicit causal relationships between transitions that have not been observed consecutively. The discovered PN fires exactly the sequences $S_{i}$ from $M_{0}$ and may eventually accept exceeding iterative sub-sequences, which correspond to the behaviour inherent to $\mathrm{PN}$ with repetitive components.

Implementation and tests revealed accuracy and efficiency of the method when complex $P N$ structures were addressed. Current research addresses the problem of PN discovery from incomplete observed sequences.

\section{REFERENCES}

[1] M. E. Gold, "Language identification in the limit", Information and Control, 10(5), pp. 447-474, 1967

[2] D. Angluin, "Queries and Concept Learning", Machine Learning, vol. 2, pp. 319-342, 1988

[3] M. Meda-Campana, A. Ramirez-Treviño, and E. LopezMellado, "Asymptotic identification of discrete event systems", in Proc. of the $39^{\text {th }}$ IEEE Conf. on Decision and Control, pp. 22662271,2000

[4] M. Meda-Campana and E. López-Mellado, "Identification of concurrent discrete event systems using Petri nets", in Proc. of the $17^{\text {th }}$ IMACS World Congress on Computational and Applied Mathematics, pp. 11-15, 2005

[5] M.P. Cabasino, A. Giua, C. Seatzu, "Identification of Petri nets from knowledge of their language," Discrete Event Dynamic Systems, Vol. 17, No. 4, pp. 447-474, 2007

[6] M. P. Cabasino, A. Giua, and C. Seatzu, "Linear programming techniques for the identification of place/transition nets", in Proc. of the $47^{\text {th }}$ IEEE Conf. on Decision and Control, pp. 514520, 2008

[7] M. Dotoli, M. Pia Fanti, A. M. Mangini, and W. Ukovich, "Identification of the unobservable behaviour of industrial automation systems by Petri nets", Control Engineering Practice, 19( 9), pp. 958-966, 2011

[8] S. Klein, L. Litz, J.-J. Lesage, "Fault detection of discrete event systems using an identification approach", in Proc. of the $16^{\text {th }}$ IFAC world Congress, 6 pages, 2005
[9] M. Roth, S. Schneider, J.-J. Lesage, and L. Litz, "Fault detection and isolation in manufacturing systems with an identified discrete event model", International Journal of Systems Science, 43(10), pp. 1826-1841, 2012

[10] A.P. Estrada-Vargas, E. Lopez-Mellado, and J.-J. Lesage, "Identification of partially observable discrete event manufacturing systems", in Proc. of the $18^{\text {th }}$ IEEE Conf. on Emerging Technologies \& Factory Automation, pp. 1-7, 2013

[11] A.P. Estrada-Vargas, J-J. Lesage, E. López-Mellado "A Stepwise Method for Identification of Controlled Discrete Manufacturing Systems". Int. Journal of Computer Integrated Manufacturing. Pub. on-line: Jan, 27, 2014. ISSN: 0951-192X pp.1-13

[12] A.P. Estrada-Vargas, E. Lopez-Mellado, and J.-J. Lesage, "A comparative analysis of recent identification approaches for discrete event systems", Mathematical Problems in Engineering, vol. 2010, 2010

[13] M. P. Cabasino, P. Darondeau, M. P. Fanti, and C. Seatzu, "Model identification and synthesis of discrete-event systems", Contemporary Issues in Systems Science and Engineering, IEEE/Wiley Press Book Series 2013

[14] R. Agrawal, D. Gunopulos, and F. Leymann, "Mining Process Models from Workflow Logs", Lecture Notes in Computer Science, Vol. 1377, pp. 469-483, 1998

[15] J. E. Cook, Z. Du, C. Liu, and A. L. Wolf, "Discovering models of behavior for concurrent workflows", Computers in industry, 53(3), pp. 297-319, 2004

[16] W. Van der Aalst, T. Weijters, and L. Maruster, "Workflow mining: Discovering process models from event logs", IEEE Trans. On Knowledge and Data Engineering, 16(9), pp. 11281142,2004

[17] L. Wen, J. Wang, and J. Sun, "Detecting implicit dependencies between tasks from event logs", Lecture Notes in Computer Science, Vol. 3841, pp 591-603, 2006

[18] D. Wang, J. Ge, H. Hu, and B. Luo, "A new process mining algorithm based on event type", in Proc. of the $9^{\text {th }}$ IEEE Conf. on Dependable Autonomic and Secure Computing, pp. 1144-1151, 2011

[19] D. Wang, J. Ge, H. Hu, B. Luo, and L. Huang, "Discovering process models from event multiset", Expert Systems with Applications, 39(15), pp. 11970-11978, 2012.

[20] W. M. Van der Aalst, "Discovery, Conformance and Enhancement of Business Processes", 368 pages, Springer, 2011

[21] PIPE 2: Platform Independent Petri net Editor 2, http://pipe2.sourceforge.net/ 